\title{
A VARIATIONAL METHOD TO STUDY THE ZAKHAROV EQUATION
}

\author{
ARUN KUMAR
}

Received 7 February 2005 and in revised form 4 August 2005

A variational method given by Ritz has been applied to the Zakharov equation to construct an analytical solution. The solution of Zakharov equation gives a good description of both linear and nonlinear evolutions of instabilities generated in waves due to modulation. The spatially periodic trial function is chosen in the form of combination of Jacobian elliptic functions with the dependence of its parameters subject to optimization. This Zakharov equation is reduced to nonlinear Schrödinger equation in the static limit.

\section{Introduction}

The Zakharov equation [14] can be formulated as the envelope equation of a dispersive wave system [12], which is almost monochromatic and highly nonlinear. The Zakharov equation has various applications in physics in a theory of deep-water waves [8], communication [6], and nonlinear pulse propagation in fibers [2]. In the static limit of the field, the Zakharov equation is reduced to nonlinear Schrödinger equation [7].

The inverse scattering technique discovered by Zabusky and Kruskal [13] is a powerful tool for exact solution of integrable equations, like the KdV equation and the nonlinear Schrödinger equation. For nonintegrable equations, like the Zakharov equation, the Ritz variational method may be used for approximative solution.

Anderson [2] first explains the nonlinear pulse propagation in optical fibers as governed by the nonlinear Schrödinger equation using variational approach. He used secanttype trial function. But the main shortcoming of the use of trial function was the inability to account for changes in pulse shape. Then he used Gaussian type of trial function in which trial function amplitude, width, and frequency of the function may vary, but the Gaussian shape was assumed inherently preserved. The Gaussian shape pulse [5] $\Psi(t, x)=A(x) \exp \left[-t^{2} / 2 a^{2} x+i b x t^{2}\right]$ will reproduce the exact solution from variational problem in linear limit.

\section{Variational formulation of Zakharov equation}

The Zakharov equations given by

$$
i E_{t}+E_{x x}-n E=0, \quad n_{t t}-n_{x x}-|E|_{x x}^{2}=0
$$


are the coupled partial differential equations. Here, $E(x, t)$ is the slowly varying envelope of the high-frequency field, and $n$ is the density of the media or ions in media. These Zakharov equations can be approximated by nonlinear Schrödinger equation $[5,4]$.

The Zakharov equation (2.1) can be formulated as the variational problem corresponding to Lagrangian

$$
L(t)=\int_{-\lambda / 2}^{\lambda / 2} \wp(x, t) d x
$$

Lagrangian $\wp(x, t)$ is given by

$$
\wp(x, t)=\frac{i}{2}\left[E^{*} E_{t}-c \cdot c\right]-\left|E_{x}\right|^{2}+\frac{1}{2}\left[\left\{u_{t}-\left(E \cdot E^{*}\right)\right\}^{2}-u_{x}^{2}\right],
$$

where

$$
u_{t}=n+|E|^{2} .
$$

The asterisks and $c \cdot c$ denote the complex conjugate, and the limit of integration is the periodicity length $\lambda$, which will later be assumed as constant.

Now, first we will reproduce the Zakharov equations by using Euler-Lagrange equations:

$$
\begin{aligned}
& \frac{\partial \wp}{\partial E}-\frac{d}{d t} \frac{\partial \wp}{\partial E_{t}}-\frac{d}{d x} \frac{\partial \wp}{\partial E_{x}}=0 \\
& \frac{\partial \wp}{\partial u}-\frac{d}{d t} \frac{\partial \wp}{\partial u_{t}}-\frac{d}{d x} \frac{\partial \wp}{\partial u_{x}}=0
\end{aligned}
$$

On substituting the values from (2.3) in (2.5), we obtain the Zakharov equation (2.1) which shows that the selection of Lagrangian density (2.2) is compatible.

We employ the Ritz variational principle to the action integral $S(t)=\int L(t) d t$ with respect to time-dependent parameters of the trial function which admits:

(1) the shape of an unmodulated wave with a sinusoidal disturbance,

(2) provide spatial periodicity of the Lagrangian with period $\lambda$.

So, these features are provided by $[10,11]$

$$
E(x, t)=A(t)[\operatorname{dn}(z ; \beta)+\beta c n(z ; \beta)] \times \exp \left[i\left\{\frac{k z}{\alpha}+c \cos \left(\frac{2 \pi z}{\alpha \lambda}\right)+\phi\right\}\right] .
$$

The time-dependent functions, independent from one another, are $A, \beta, c, \phi, \alpha, x_{0}$, and $k$.

Here, $d n(z ; \beta)$ and $c n(z ; \beta)$ are the Jacobian elliptic functions for $z=\alpha\left(x-x_{0}\right)$ and $\alpha=4 K(\beta) / \lambda$, and $K(\beta)$ is the complete elliptic integral of first kind. For small parameters $\beta$ and $c$, one obtain from (2.6)

$$
E(x, t)=A(t)[1+(\beta+i c) \cos z] \times \exp i\left[\frac{k z}{\alpha}+\phi\right] .
$$

Equation (2.7) describes envelops of a finite amplitude wave with (wave number $k$ ) slightly modulated by a plane wave with wave number $\alpha$. We have seen that Zakharov 
equation is reduced to NLS equation in the static limit $n=-|E|^{2}[7,9]$. So the total Lagrangian (2.3) can be written as

$$
\wp=\wp_{\mathrm{NLS}}+\wp_{1}
$$

where

$$
\wp_{\mathrm{NLS}}=\frac{i}{2}\left[E^{*} E_{t}-c \cdot c\right]-\left|E_{x}\right|^{2}+\frac{1}{2}\left(E \cdot E^{*}\right)^{2}
$$

(Euler-Lagrange equation reproduces the NLS equation from $\wp_{\mathrm{NLS}}$ ) and

$$
\wp_{1}=-\frac{1}{2}\left(E^{*} E\right)^{2}+\frac{1}{2}\left[\left\{u_{t}-\left(E \cdot E^{*}\right)\right\}^{2}-u_{x}^{2}\right]
$$

is the additional part. So the action integral for Zakharov equation becomes

$$
S=\int\left(\wp_{\mathrm{NLS}}+\wp_{1}\right) d t
$$

Now we assume trial function for $\wp_{1}$ as

$$
u=B(t) E E^{*} .
$$

Now we substitute our trial function in (2.2) after changing $\alpha\left(x-x_{0}\right)=z$ and $k(t)=$ $V / 2$. We get

$$
\begin{aligned}
L= & \alpha^{-1} A^{2}\left[c_{t} I_{1}+\left(-\phi_{t}-\frac{V x_{0, t}}{2}+\frac{V^{2}}{4}\right) I_{2}-\frac{4 \pi^{2} c^{2}}{\lambda^{2}} I_{3}\right]-\alpha A^{2} I_{4}+\frac{1}{2} \alpha^{-1} A^{4} I_{5} \\
& +\alpha^{-1} A^{2}\left(\frac{\left(B_{t}-1\right)^{2}}{2} A^{2}-1\right) I_{5}-\frac{B^{2}}{2} \alpha A^{4} I_{6},
\end{aligned}
$$

where

$$
\begin{aligned}
& I_{1}=\int_{-2 k}^{2 k} d z[d n(z ; \beta)+\beta c n(z ; \beta)]^{2} \cos \left(\frac{\pi z}{2 K}\right)=\frac{2 \pi^{2} \operatorname{sgn}(\beta)}{K \sinh \left(\pi K^{\prime} / 2 K\right)}, \\
& I_{2}=\int_{-2 k}^{2 k} d z[d n(z ; \beta)+\beta c n(z ; \beta)]^{2}=4\left[2 E-\beta^{\prime 2} K\right]=4 C_{1}, \\
& I_{3}=\int_{-2 k}^{2 k} d z[d n(z ; \beta)+\beta c n(z ; \beta)]^{2} \sin ^{2}\left(\frac{\pi z}{2 K}\right)=2\left[2 E-\beta^{\prime 2} K-\frac{\pi^{2}}{\left(K \sinh \left(\pi K^{\prime} / 2 K\right)\right)}\right], \\
& I_{4}=\int_{-2 k}^{2 k} d z\left[\frac{d}{d z}\{d n(z ; \beta)+\beta c n(z ; \beta)\}\right]^{2}=\frac{4}{3}\left[\left(1+\beta^{2}\right) E-\left(1-\beta^{2}\right) K\right]=\frac{4 C_{2}}{3}, \\
& I_{5}=\int_{-2 k}^{2 k} d z[d n(z ; \beta)+\beta c n(z ; \beta)]^{4}=\frac{4}{3}\left[8\left(1+\beta^{2}\right) E-\left(5+3 \beta^{2}\right)\left(1-\beta^{2}\right) K\right]=\frac{4 C_{3}}{3}, \\
& I_{6}=\int_{-2 k}^{2 k} d z\left[\frac{d}{d z}\{d n(z ; \beta)+\beta c n(z ; \beta)\}^{2}\right]^{2}=\frac{4 C_{4}}{3} .
\end{aligned}
$$


Here, assumptions $\alpha=4 K / \lambda$ and

(1) $D=-2 d E / d\left(\beta^{2}\right)=(K-E) / \beta^{2}>0$,

(2) $B=-2 d K / d\left(\beta^{2}\right)=\left(E-\beta^{\prime 2} K\right) /\left(\beta^{2} \beta^{\prime 2}\right)>0$,

(3) $C=-2 d B / d\left(\beta^{2}\right)=\left[(2-\beta)^{2} K-2 E\right] / \beta^{4}>0, \beta^{\prime}=1-\beta^{2}$,

are used to calculate above integrals. $C_{1}, C_{2}, C_{3}$, and $C_{4}$ are the quantities in fraction and are the combination of complete elliptic integrals $K, E$, and their argument $\beta$. So the action integral $S$ becomes

$$
\begin{aligned}
& S=\int L d t \\
&=\int d t\left[-\frac{\lambda A^{2}}{K} C_{1}\left\{\phi_{t}-\frac{V}{2} x_{0 t}+\frac{V^{2}}{4}+\frac{2 \pi^{2} c^{2}}{\lambda^{2}}\right\}-\frac{\pi^{2} \lambda A^{2} \operatorname{sgn} \beta c_{t}}{\left[2 K^{2} \sinh \left(\pi K^{\prime} / 2 K\right)\right]}\right. \\
&\left.+\frac{2 \pi^{4} A^{2} c^{2}}{\lambda K^{2} \sinh \left(\pi K^{\prime} / 2 K\right)}-\frac{16 K A^{2} C_{2}}{3 \lambda}+\frac{\lambda C_{3} A^{4}\left(B_{t}-1\right)^{2}}{6 K}-\frac{8 B^{2} K A^{4} C_{4}}{3 \lambda}\right] .
\end{aligned}
$$

The variable $x_{0}$ is absent in the action integral except for the combination $\phi-V x_{0} / 2$, and hence all trajectories $x_{0}(t)$ are equivalent provided that $\phi(t)$ is approximately shifted.

Now we proceed to study the variational equations for the parameters $A, \beta, c, B$, and $\phi$ with the help of Euler-Lagrange equations. Then, our system of equations, which follows from action integral, consists of the following conservation laws and Euler-Lagrange equations [1].

(1) Integrated variation $\varphi$ equation (Plasmon number $N$ conservation):

$A^{2}\left[\frac{2 E}{K}-\left(1-\beta^{2}\right)\right]=\frac{N}{\lambda} \quad$ (where $N$ is constant called Plasmon number).

(2) The Hamiltonian $H$ :

$$
H-\frac{N V^{2}}{4}=U+\frac{c^{2}}{2 M}+\frac{\lambda A^{4} C_{3} B_{t}^{2}}{6 K}+\frac{8 \beta^{2} K A^{4} C_{1}}{3},
$$

where $U=16 K A^{2} C_{2} / 3 \lambda-\lambda A^{4} C_{3} / 6 K$, and time-dependent mass

$$
M=\left(\frac{\lambda}{4 \pi^{2}}\right)\left[\frac{N}{\lambda}-\frac{\pi^{2} A^{2}}{\left\{K^{2} \sinh \left(\pi K^{\prime} / K\right)\right\}}\right]^{-1} .
$$

(3) Variation $c$ equation:

$$
\frac{\partial Q}{\partial t}=\frac{c}{M}
$$

where $Q$, which plays the role of a generalized coordinate, is the amplitude of the first Fourier mode of $\left|E^{2}\right|^{2}$ and

$$
Q=\frac{\pi^{\lambda} A^{2} \operatorname{sgn} \beta}{\left[2 K^{2} \sinh \left(\pi K^{\prime} / 2 K\right)\right]} .
$$




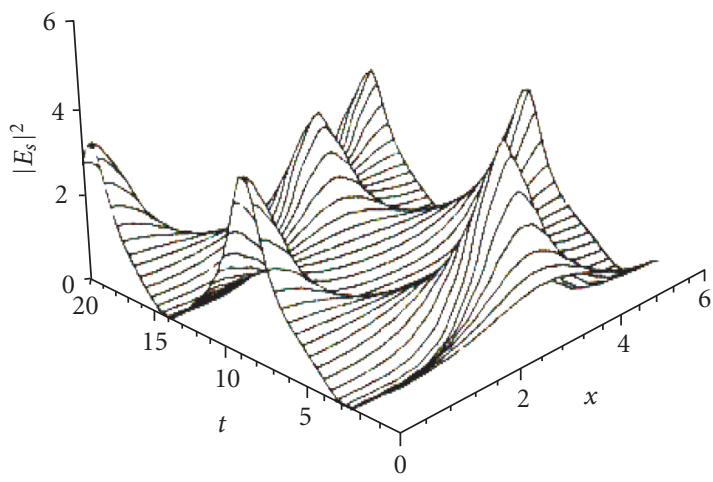

Figure 3.1. Numerical solution to the Zakharov equations in static limit. The initial conditions are $A=1, \alpha=1.2, \beta=c=0.1$, and $V=0$.

(4) Variation $A$ equation:

$$
N \phi_{t}+Q c_{t}=\frac{N V^{2}}{4}-\frac{c^{2}}{2 M}-\frac{16 K A^{2} C_{2}}{3 \lambda}+\frac{\lambda C_{3} A^{4}\left(B_{t}-1\right)^{2}}{3 K}-\frac{32 K A^{4} C_{4}}{3 \lambda} .
$$

(5) Variation $B$ equation:

$$
\frac{\lambda C_{3} A^{4} B_{t t}}{3 K}+\frac{32 K A^{4} C_{4} B}{3 \lambda}=0 .
$$

Equations (2.16) to (2.19) form a closed system, while (2.21) and (2.22) contribute to the phase $\phi$ and parameter $B$ only. The variational criterion either leaves these functions or $\phi$ and $B$ undetermined. The equation (2.16) to (2.19) may be solved by a single nonelementary quadrature.

\section{A direct numerical method}

Zakharov equations

$$
\begin{gathered}
i E_{t}+E_{x x}-n E=0, \\
n_{t t}-n_{x x}-|E|_{x x}^{2}=0
\end{gathered}
$$

can directly be solved by finite difference scheme [3], where $E(x, t)$ is the slowly varying envelope of the high-frequency field and is given by $E_{s}(x, t)=A(t)[1+(\beta+i c) \cos z] \times$ $\exp i[k z / \alpha+\phi]$. The initial conditions in static limit are $A=1, \alpha=1.2, \beta=c=0.1$, and $V=0, \alpha\left(x-x_{0}\right)=z, x_{0}=0$. A specific Crank-Nicholson scheme of finite difference with step length $h=0.4$ in $x$, and $l=1$ in $t$ (time), is applied for first part, and a simple finite difference scheme is used for second part of the equation with same step lengths. The solution is given below.

Figure 3.2 shows the example that corresponds to $H-N V^{2} / 4>U(\beta=0)$. These results, with $\beta=c=0.1$, can be compared with the outcome from the numerical integration 


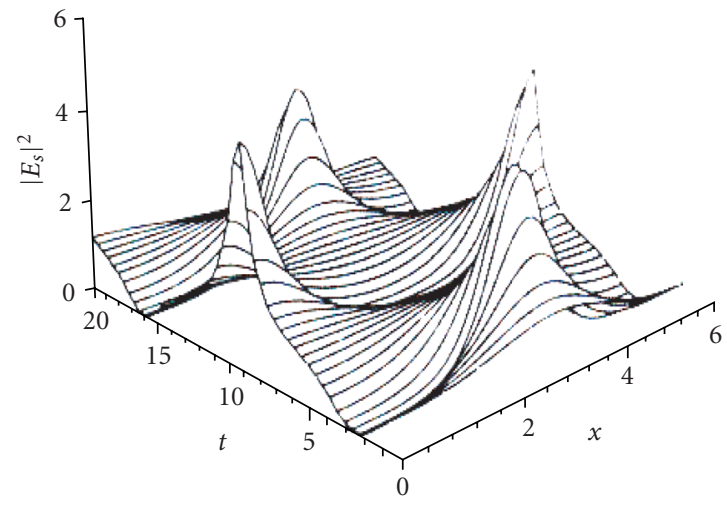

Figure 3.2. Solution to the theoretical model (2.16)-(2.19). The initial conditions are $A=1, \alpha=1.2$, $\beta=c=0.1, V=0$, and $\varphi=0$.

of Zakharov equations shown in Figure 3.1. In particular, the value of maximum fields and the exchange of energy between the first two modes closely follows the variational method.

\section{Conclusion}

We applied Ritz variational principle based on the Zakharov-Lagrangian to solve the Zakharov equation, which may be a model for both linear and nonlinear evolution of some instabilities in a wave system or flow. Spatial variance of trial function was assumed a priori, while time dependence of its parameters was subject to optimization. The crucial point, finding an appropriate trial function, was solved by introducing the variability to parameters of a stationary solution of the Zakharov equation. We chose the solution in the form of a combination of Jacobian elliptic functions. The results of the theoretical model compare well the numerical solution to Zakharov equation.

\section{Acknowledgment}

This work is partially supported by CSIR of India.

\section{References}

[1] H. Airault, H. P. McKean, and J. Moser, Rational and elliptic solutions of the Korteweg-de Vries equation and a related many-body problem, Comm. Pure Appl. Math. 30 (1977), no. 1, 95148.

[2] D. Anderson, Variational approach to nonlinear pulse propagation in optical fibers, Phys. Rev. A 27 (1983), no. 6, 3135-3145.

[3] Q. S. Chang, B. L. Guo, and H. Jiang, Finite difference method for generalized Zakharov equations, Math. Comp. 64 (1995), no. 210, 537-553, S7-S11.

[4] P. L. Christiansen, J. C. Eilbeck, V. Z. Enolskii, and N. A. Kostov, Quasi-periodic solutions of the coupled nonlinear Schrödinger equations, Proc. Roy. Soc. London Ser. A 451 (1995), no. 1943, $685-700$. 
[5] - Quasi-Periodic and Periodic solutions for coupled nonlinear Schrödinger equations of Manakov type, Proc. Roy. Soc. London Ser. A 456 (2000), no. 2001, 2263-2281.

[6] M. V. Goldman, Strong turbulence of plasma waves, Rev. Modern Phys. 56 (1984), no. 4, 709735.

[7] B. Hafizi, Nonlinear evolution equations, recurrence and stochasticity, Phys. Fluids 24 (1981), no. $10,1791-1798$.

[8] E. Kit and L. Shemer, Spatial versions of the Zakharov and Dysthe evolution equations for deepwater gravity waves, J. Fluid Mech. 450 (2002), 201-205.

[9] A. V. Porubov and D. F. Parker, Some general periodic solutions to coupled nonlinear Schrödinger equations, Wave Motion 29 (1999), no. 2, 97-109.

[10] I. M. Sigal, Nonlinear wave and Schrödinger equations: I. Instability of periodic and quasiperiodic solutions, Comm. Math. Phys. 153 (1993), no. 2, 297-320.

[11] A. O. Smirnov, Elliptic solutions of the Korteweg-de Vries equation, Mat. Zametki 45 (1989), no. 6, 66-73, 111 (Russian).

[12] F. F. Sun, Numerical Studies on Zakharov System, Master's thesis, National University, Singapore, 2003.

[13] N. J. Zabusky and M. D. Kruskal, Interaction of "Solitons" in a collisionless plasma and the recurrence of initial states, Phys. Rev. Lett. 15 (1965), no. 6, 240-243.

[14] V. E. Zakharov and A. B. Shabat, Exact theory of two-dimensional self-focusing and onedimensional self-modulation of waves in nonlinear media, Ž. Èksper. Teoret. Fiz. 61 (1971), no. 1, 118-134, Soviet Physics JETP 34 (1972), no. 1, 62-69.

Arun Kumar: Department of Mathematics, Government College, Kota (Raj)-324001, India E-mail address: arunkr71@sancharnet.in 


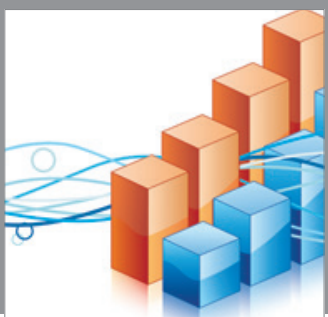

Advances in

Operations Research

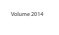

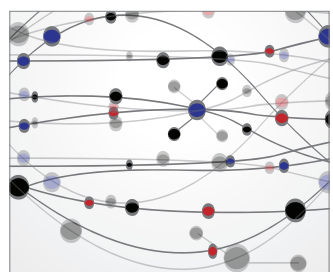

\section{The Scientific} World Journal
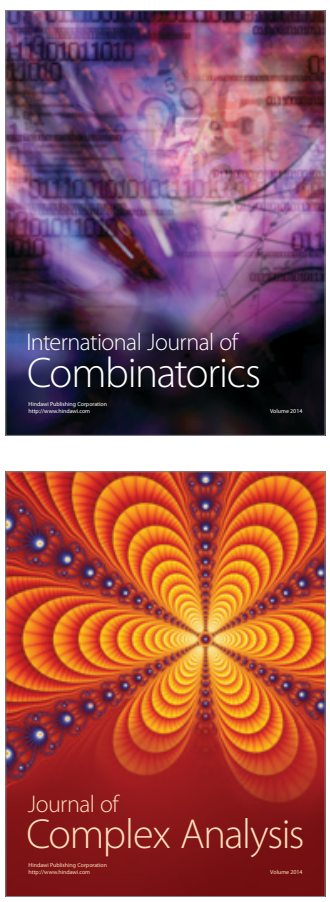

International Journal of

Mathematics and

Mathematical

Sciences
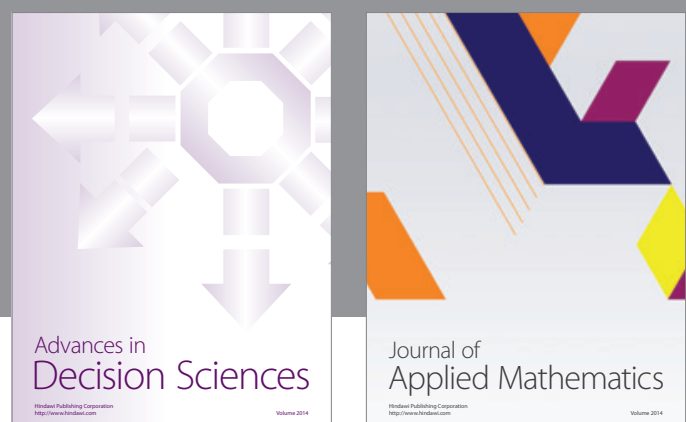

Journal of

Applied Mathematics
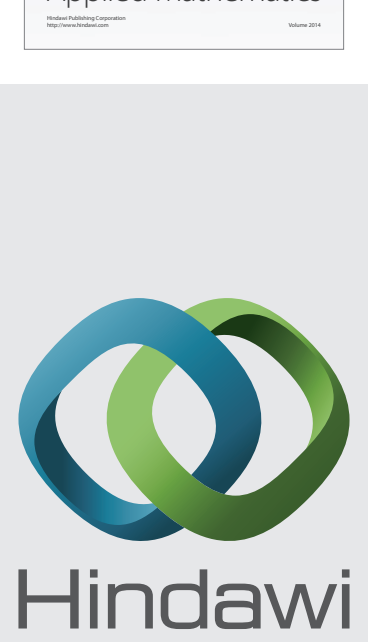

Submit your manuscripts at http://www.hindawi.com
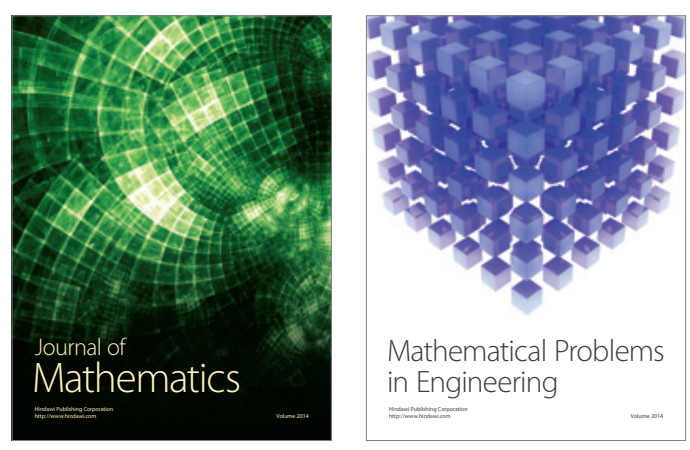

Mathematical Problems in Engineering
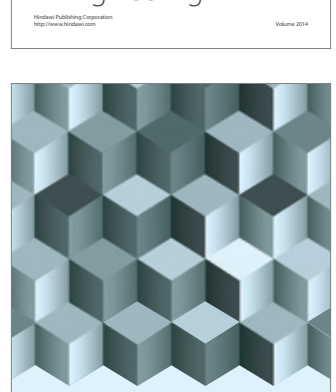

Journal of

Function Spaces
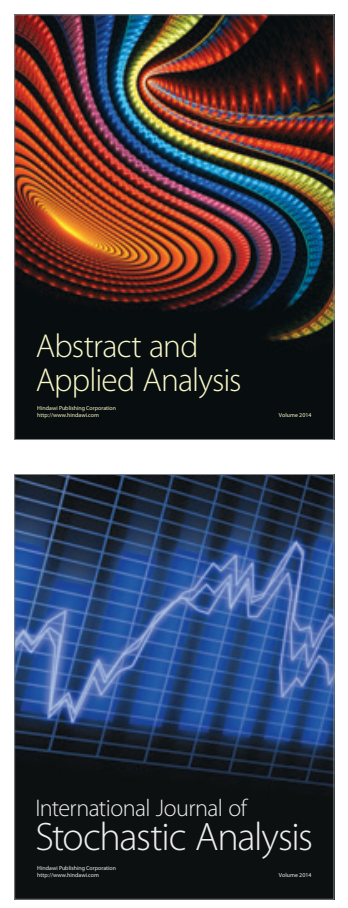

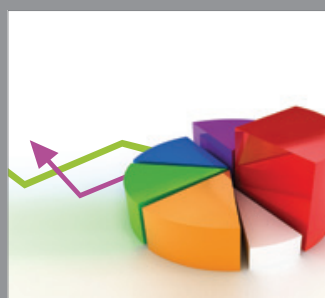

ournal of

Probability and Statistics

Promensencen
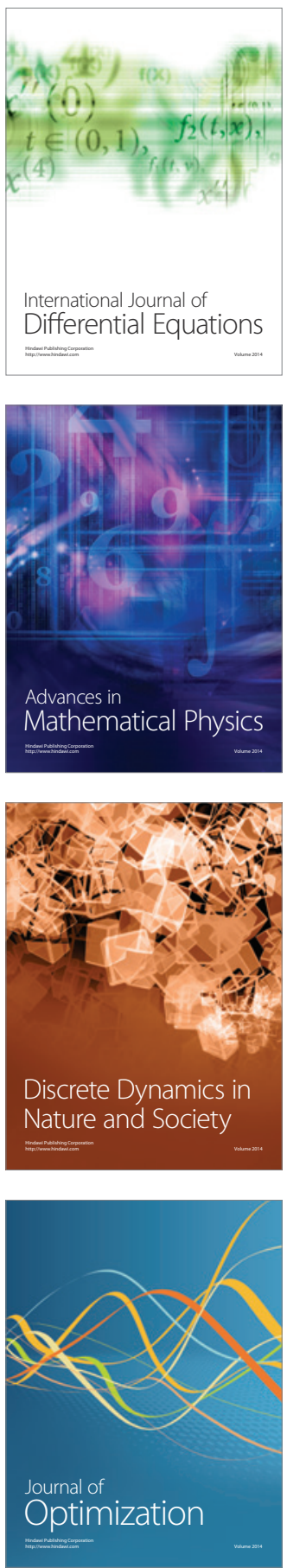